\title{
Microscopic Model of Charge Carrier Transfer in Complex Media
}

\author{
O.Bénichou $^{\mathrm{a}}$, J.Klafter ${ }^{\mathrm{b}}$, M.Moreau $^{\mathrm{a}}$, and G.Oshanin ${ }^{\mathrm{ac} *}$ \\ a Laboratoire de Physique Théorique de la Matière Condensée, \\ Université Paris 6, 4, Place Jussieu, 75252 Paris, France \\ bSchool of Chemistry, \\ Tel Aviv University, Tel Aviv 69978, Israel
}

${ }^{\mathrm{c}}$ Department of Inhomogeneous Condensed Matter Theory, Max-Planck-Institut für Metallforschung, Heisenbergstrasse 3, D-70569 Stuttgart, Germany

We present a microscopic model of a charge carrier transfer under an action of a constant electric field in a complex medium. Generalizing previous theoretical approaches, we model the dynamical environment hindering the carrier motion by dynamic percolation, i.e., as a medium comprising particles which move randomly on a simple cubic lattice, constrained by hard-core exclusion, and may spontaneously annihilate and reappear at some prescribed rates. We determine analytically the density profiles of the "environment" particles, as seen from the stationary moving charge carrier, and calculate its terminal velocity, $V_{c}$, as the function of the applied field and other system parameters. We realize that for sufficiently small external fields the force exerted on the carrier by the "environment" particles shows a viscous-like behavior and define an analog of the Stokes formula for such dynamic percolative environments. The corresponding friction coefficient is also derived. We show that the density profile of the environment particles is strongly inhomogeneous: In front of the stationary moving charge carrier the density is higher than the average density, $\rho_{s}$, and approaches it as an exponential function of the distance from the carrier. Behind the carrier the local density is lower than $\rho_{s}$ and the approach towards $\rho_{s}$ may proceed differently depending on whether the particles number is or is not explicitly conserved.

\section{INTRODUCTION}

The percolation concept has been a key notion for understanding transport and conduction processes in a wide range of complex disordered media. A few stray examples are ionic conduction in polymeric, amorphous or glassy ceramic electrolytes, diffusion in biological tissues and permeability of disordered membranes 1234 .

Most of physical situations studied so far (see Refs. [1234] and references therein) concern systems in which complex disordered environment can be considered as "frozen"; that is, the random environment in which a given transfer process takes place does not

${ }^{*}$ Corresponding author. e-mail: oshanin@lptl.jussieu.fr. 
change in time. This is certainly the case in many instances, but it is not true in general. In fact, there are many experimental systems in which the static percolation picture is not valid since the structure of the host material reorganizes itself on a time scale comparable to that at which the transfer itself occurs. Such a reorganization happens, namely, in certain biomembranes [5], solid protonic conductors [6, oil-continuous microemulsions 78 8910 or polymer electrolytes [11 1213 .

In particular, ionic transport across a biomembrane, such as, e.g., gramicidin- $A$, proceeds by the motion of ions through molecular channels along which they encounter potential barriers whose heights fluctuate randomly in time. The fluctuations of potential barriers may impede the transport constituting an important controlling factor [5]. In the case of protonic conduction by the Grotthus mechanism [6], site-to-site hopping of charge carriers takes place between neighboring $\mathrm{H}_{2} \mathrm{O}$ or $\mathrm{NH}_{3}$ groups that have a favorable relative orientation. Here, the structural host-reorganization process interacting with the carrier motion occurs due to thermally activated rotation of the $\mathrm{H}_{2} \mathrm{O}$ or $\mathrm{NH}_{3}$ groups. In a similar fashion, within oil-continuous microemulsions, the charge transport proceeds by charge being transferred from one water globule to another, as globules approach each other in their Brownian motion [78910]. Last but not least, in polymer electrolytes, such as, e.g. polyethylene oxide complexed non-stoichiometrically with the ionic salt $N a S C N$, the $\mathrm{Na}^{+}$ions are largely tetrahedrally coordinated by polyether oxygens, but at the same time that $\mathrm{Na}^{+}$ions hop from one fourfold coordination site to another, the oxygens themselves, along with the polymeric backbone, undergo large-amplitude wagging and even diffusive motion 111213 .

Clearly, all these examples involve two characteristic time scales: the typical time $\tau$ between two successive hops of the charge carrier and a typical renewal time $\tau^{*}$ of the environment itself; that is, the time at which the host complex medium re-organizes itself sufficiently enough to provide a new set of available pathways for charge carrier transfer. Accordingly, the conventional static percolation picture can be strictly valid when only the characteristic time $\tau^{*}$ gets infinitely large. Only in this case one may expect an anomalous large-scale dynamics. On contrary, when $\tau^{*}$ is finite, dynamic percolation picture has to be applied. In this situation, one encounters quite a different behavior when compared to the random environments with quenched disorder. As a result, one expects an Ohmictype or Stokes-type linear velocity-force relation for the carrier's terminal velocities as a function of the applied field, in contrast to the threshold behavior and anomalous dynamics predicted by the conventional static percolation theory.

The prefactor in the linear velocity-force relation may depend, however, in a non-trivial way on the system's parameters and this dependence constitutes the main challenge for the theoretical analysis here. On the other hand, we note that in the above mentioned examples of the dynamic percolative environments quite different physical processes are responsible for the time evolution of the host medium. Consequently, one expects that the prefactor in the Stokes-type velocity-force relation should also be dependent on the precise mechanism which underlies the temporal re-organization of the environment.

Theoretical modeling of charge carrier transfer in dynamic percolative environments has followed basically two different lines of thought. Early models of dynamic percolation 1415. described the random environment within the framework of a standard bondpercolation model allowing the strength of each bond to fluctuate in time between zero 
and some finite value. In this approach, the dynamics of the host medium [1415] was accounted for by a series of instantaneous renewal events. These events were assumed to occur at random times, chosen from a renewal time distribution. In the renewal process the positions of all unblocked bonds are being reassigned, such that after each renewal event a carrier sees a newly defined network. This approach is thus characterized by a global dynamical disorder without global conservation laws and correlations, since the entire set of random hopping rates is renewed independently of the previous history. Another model characterized by a local dynamical disorder has been proposed in Refs. [16] and [17], and subsequently generalized to the non-Markovian case in Ref. 18. The difference of this model in regard to the previous one is that here the hopping rates at different sites fluctuate independently of each other. That is, states of individual bonds, rather than that of the whole lattice change in the renewal events. To describe the dynamical behavior in the local dynamical disorder case, a dynamical mean-field theory has been proposed [16 17], based on the effective medium approximation introduced for the analysis of random walks on lattices with static disorder [19. Subsequently, this model has been generalized to include the possibility of multistate transformations of the dynamically random complex medium [20]. More recently, several exactly solvable one-dimensional models with global and local dynamical disorder have been discussed [21.

The second approach to the problem emerged within the context of ionic conductivity in superionic solids. Here, the dynamical percolative environment has been considered as a multicomponent mixture of mobile species in which one or several neutral components block the carrier component [22]. In particular, such a situation can be observed in a superionic conductor $\beta^{\prime \prime}$-alumina, doped with two different ionic species (e.g. $\mathrm{Na}^{+}$and $\mathrm{Ba}^{2+}$ ), where small $\mathrm{Na}^{+}$ions are rather mobile, while the larger $\mathrm{Ba}^{2+}$ ions move essentially slower and temporarily block the $\mathrm{Na}^{+}$ions. Contrary to the previous line of thought, the dynamics of such a percolative environment has essential correlations, generated by hard-core exclusion interactions between the species involved. Moreover, a salient feature of these situations is that here the total number of the particles involved is conserved, i.e., dynamics of the environment is globally constrained by a certain "conservation law". The impact of this property on charge carrier transfer will become clearer as we proceed.

Further on, in Ref. 22] the frequency-dependent ionic conductivity of the light species has been analyzed combining a continuous time random walk approach for the dynamical problem with an effective medium approximation describing the frozen environment of slow species. Next, as an explanation of the sharp increase of electrical conductivity transition in water-in-oil microemulsions when the volume fraction of water is increased towards a certain threshold value, in Refs. [8] and [9] it has been proposed that the charge carriers are not trapped in the finite water clusters, but rather a charge on a water globule can propagate by either hopping to a neighboring globule, when they approach each other, or via the diffusion of the host globule itself. This picture has been interpreted in terms of a model similar to that employed in Ref.[22, with the only difference being that here the "blockers" of Ref. 22] play the role of the transient charge carriers. In the model of Refs. [8] and [9], in which the host dynamics is influenced by spatial correlations and conservation of the number of the water globules involved, the conductivity depends on the rate of cluster rearrangement. Lastly, a similar problem of a carrier diffusion in an environment created by mobile hard-core lattice-gas particles has been analyzed in Ref. [23] by using 
the developed dynamic bond percolation theory of Refs. 14] and [15].

In this paper, we pursue our previous analysis of the charge carrier transfer in complex media [24] considering a generalize dynamic percolation model which shares common features with both bond-fluctuating models of Refs. 14151617182021 and those involving mobile blockers of Refs. 2223 . The system we consider consists of a host lattice, which here is a regular cubic lattice whose sites support at most a single occupancy, hard-core "environment" particles, and a single, hard-core charge carrier particle subject to external electric field. The "environment" particles move on the lattice by performing a random hopping between the neighboring lattice sites, which is constrained by the hard-core interactions, and may disappear from and re-appear (renewal processes) on the empty sites of the lattice with some prescribed rates. Contrary to the environment particles the charge carrier can not disappear spontaneously, and is subject to a constant external force $\vec{E}$. Hence, the carrier performs a biased random walk, which is constrained by the hard-core interactions with the "environment" particles. In consequence, on may consider the carrier as some "probe" designed to measure the response of the percolative environment to the internal perturbation or, in other words, the frictional properties of such a dynamical environment.

Now, a salient feature of our model, which makes it different to the previously proposed models of dynamic percolation, is that here the interaction between "environment" particles and the carrier are included, such that the latter may influence the dynamics of the environment. This results, as we proceed to show, in the emergence of complicated density profiles of the "environment" particles around the carrier. These profiles, as well as the terminal velocity $V_{c}$ of the carrier, are determined here explicitly, in terms of an approximate approach of Ref. [25], which is based on the decoupling of the triple carrier-particle-particle correlation functions into the product of pair correlations.

We realize that the "environment" particles tend to accumulate in front of the driven charge carrier creating a "traffic jam", which impedes its motion. The density profiles around the carrier are asymmetric: the local density of the "environment" particles in front of the carrier is higher than the average and approaches the average value as an exponential function of the distance from the carrier. The characteristic length and the amplitude of the density relaxation function are calculated explicitly. On the other hand, behind the carrier, i.e. in its "wake", the local density is lower than the average. We find that, surprisingly, the functional form of the density profile might be very different depending on the condition whether the number of particles in the percolative environment is explicitly conserved or not; as a matter of fact, the local density behind the carrier may tend to the average value either as an exponential or even as an algebraic function of the distance, revealing in the latter case especially strong memory effects and strong correlations between the particle distribution in the environment and the carrier position. Further on, we find that the terminal velocity of the carrier particle depends explicitly on the excess density in the "jammed" region in front of the carrier, as well as on the "environment" particles density past the carrier. Both, in turn, are dependent on the magnitude of the velocity, as well as on the rate of the renewal processes and the rate at which the "environment" particles can diffuse away from the carrier. The interplay between the jamming effect of the environment, produced by the carrier particle, and the rate of its homogenization due to diffusive smoothening and renewal processes, man- 
ifests itself as a medium-induced frictional force exerted on the carrier, whose magnitude depends on the carrier velocity. As a consequence of such a non-linear coupling, in the general case, (i.e. for arbitrary rates of the renewal and diffusive processes), $V_{c}$ can be found only implicitly, as the solution of a non-linear equation relating $V_{c}$ to the system parameters. This equation simplifies considerably in the limit of small applied external fields $\vec{E}$ and we find that the force-velocity relation to the field becomes linear. This implies that the frictional force exerted on the carrier particle by the environment is viscous. This linear force-velocity relation can be therefore interpreted as the analog of the Stokes formula for the dynamic percolative environment under study; in this case, the carrier velocity is calculated explicitly as well as the corresponding friction coefficient. Assuming that the Einstein relation between the carrier mobility and its diffusion coefficient holds [26], we then estimate the self-diffusion coefficient of the carrier in absence of external field. We show that when only diffusive re-arrangement of the percolative environment is allowed, while the renewal processes are suppressed, the general expression for the diffusion coefficient reduces to the one obtained previously in Refs. 27] and [28]. We note that the result of Refs. 27] and [28] is known to serve as a very good approximation for the self-diffusion coefficient in hard-core lattice-gases [29].

The paper is structured as follows: In Section II we formulate the model and introduce basic notations. In Section III we write down the dynamical equations which govern the time evolution of the "environment" particles and of the carrier. Section IV is devoted to the analytical solution of these evolution equations in the limit $t \rightarrow \infty$. In Section $\mathrm{V}$ we derive explicit asymptotic results for the carrier terminal velocity in the limit of small applied external fields $\vec{E}$ and obtain the analog of the Stokes formula for such a percolative environment. Asymptotic behavior of the density profiles of the "environment" particles around the carrier is discussed in Section VI. Finally, we conclude in Section VII with a brief summary and discussion of our results.

\section{THE MODEL.}

The model for charge carrier transfer in complex media consists of a three-dimensional simple cubic lattice of spacing $\sigma$, the sites of which are partially occupied by identical hard-core "environment" particles and a single, hard-core, carrier particle. For both types of particles the hard-core interactions prevent multiple occupancy of the lattice sites; that is, no two "environment" particles or the "carrier" and an "environment" particle can occupy simultaneously the same site, and particles can not pass through each other.

The occupation of the lattice sites by the "environment" particles is characterized by the time-dependent occupation variable $\eta(\vec{r}), \vec{r}$ being the lattice-vector of the site in question. This variable assumes two values:

$\eta(\vec{r})=\left\{\begin{array}{l}1, \text { if the site } \vec{r} \text { is occupied } \\ 0, \text { if the site } \vec{r} \text { is empty }\end{array}\right.$

Next, the dynamics of the "environment" particles is defined via the following rules The particles may, at a given rate, spontaneously disappear from the lattice, and may reappear at random positions and random time moments, which is reminiscent of the host medium dynamics stipulated in Refs. 14,15,1617182021]. These two processes will be referred to, in general, as renewal processes. 
Now, the environment particles move randomly by performing nearest-neighbor random walks constrained by the hard-core interactions, which is the main feature of the approach in Refs. 2223]. More specifically, we stipulate that any of the "environment" particles waits a time $\delta \tau$, which has an exponential probability distribution with a mean $\tau^{*}$, and then chooses from a few possibilities: (a) disappearing from the lattice at rate $g / \tau^{*}$, which is realized instantaneously, or (b) attempting to hop, at rate $l / 6 \tau^{*}$, onto one of 6 neighboring sites. The hop is actually fulfilled if the target site is not occupied at this time moment by any other particle; otherwise, the particle attempting to hop remains at its initial position, and (c) particles may re-appear on any vacant lattice site with rate $f / \tau^{*}$.

Note that, for simplicity, we assumed that the characteristic diffusion time and the renewal times of the "environment" particles are equal to each other. These times, i.e. $\tau_{d i f}$, mean creation time $\tau_{c r}$ and mean annihilation time $\tau_{a n}$ may, however, be different, and can be restored in our final results by a mere replacement $l \rightarrow l \tau^{*} / \tau_{d i f}, f \rightarrow f \tau^{*} / \tau_{c r}$ and $g \rightarrow g \tau^{*} / \tau_{a n}$.

Note also that the number of particles is not explicitly conserved in such a dynamical model of the environment due to the renewal processes. However, in the absence of attractive particle-particle interactions and external perturbations, the particles distribution on the lattice is uniform and the average occupation $\rho(t)=\overline{\eta(\vec{r})}$ of the lattice tends, as $t \rightarrow \infty$, to a constant value, $\rho_{s}=f /(f+g)$. This relation can be thought of as the Langmuir adsorption isotherm [30].

Hence, the limit $\tau_{d i f} \rightarrow \infty$ (or, $l \rightarrow 0$ ) corresponds to the ordinary site percolation model with immobile blocked sites. The limit $f, g \rightarrow 0,\left(\tau_{c r}, \tau_{a n} \rightarrow \infty\right)$, while keeping the ratio $f / g$ fixed, $f / g=\rho_{s} /\left(1-\rho_{s}\right)$, corresponds to the usual hard-core lattice-gas with the conserved particles number.

At time $t=0$ we introduce at the origin of the lattice the charge carrier, whose role is to probe the response of the environment modeled by dynamic percolation to an external perturbation. We stipulate that only the carrier out of all participating particles can not disappear from the system, and moreover, its motion is biased by some external constant force. As a physical realization, we envisage that the carrier is charged, while all other particles are neutral, and the system is exposed to constant external electric field $\vec{E}$.

The dynamics of the carrier particle is defined as follows: We suppose that the waiting time between successive jumps of the carrier has also an exponential distribution with a mean value $\tau$, which may in general be different from the corresponding waiting time of the environment particles. Attempting to hop, the carrier first chooses a hop direction with probabilities

$p_{\mu}=\exp \left[\frac{\beta}{2}\left(\vec{E} \cdot \vec{e}_{\mu}\right)\right] / \sum_{\nu} \exp \left[\frac{\beta}{2}\left(\vec{E} \cdot \vec{e}_{\nu}\right)\right]$

where $\beta$ is the reciprocal temperature, $\vec{e}_{\nu}$ (or $\vec{e}_{\mu}$ ) stand for six unit lattice vectors, $\nu, \mu=$ $\{ \pm 1, \pm 2, \pm 3\}$, connecting the carrier position with 6 neighboring lattice sites, and $\left(\vec{E} \cdot \vec{e}_{\nu}\right)$ denotes the scalar product. We adopt the convention that \pm 1 corresponds to $\pm X, \pm 2$ corresponds to $\pm Y$ while \pm 3 stands for $\pm Z$. The jump is actually fulfilled when the target lattice site is vacant. Otherwise, the carrier remains at its position. For simplicity we assume in what follows that the external field is oriented along the $X$-axis in the 
positive direction, such that $\vec{E}=(E, 0,0)$. Note also that for the choice of the transition probabilities as in Eq.(2), the detailed balance is naturally preserved.

\section{EVOLUTION EQUATIONS.}

We proceed by writing the evolution equations describing the dynamics of the system. Let $P\left(\vec{R}_{c}, \eta ; t\right)$ denote the joint probability that at time moment $t$ the charge carrier occupies position $\vec{R}_{c}$ and all "environment" particles are in configuration $\eta \equiv\{\eta(\vec{r})\}$. Next, let $\eta^{\vec{r}, \mu}$ denote particles' configuration obtained from $\eta$ by exchanging the occupation variables of the sites $\vec{r}$ and $\vec{r}+\vec{e}_{\vec{\mu}}$, i.e. $\eta(\vec{r}) \leftrightarrow \eta\left(\vec{r}+\vec{e}_{\vec{\mu}}\right)$, and $\hat{\eta}^{\vec{r}}$ be the configuration obtained from $\eta$ by changing the occupation of the site $\vec{r}$ as $\eta(\vec{r}) \leftrightarrow 1-\eta(\vec{r})$. Clearly, the first type of process appears due to random hops of the "environment" particles, while the second one stems from the renewal processes, i.e. random creation and annihilation of the "environment" particles. Then, summing up all possible events which can result in the configuration $\left(\vec{R}_{c}, \eta\right)$ or change this configuration for any other, we find that the temporal evolution of the system under study is governed by the following master equation:

$$
\begin{aligned}
& \partial_{t} P\left(\vec{R}_{c}, \eta ; t\right)=\frac{l}{6 \tau^{*}} \sum_{\mu} \sum_{\vec{r} \neq \vec{R}_{c}-\vec{e}_{\vec{\mu}}, \vec{R}_{c}}\left\{P\left(\vec{R}_{c}, \eta^{\vec{r}, \mu} ; t\right)-P\left(\vec{R}_{c}, \eta ; t\right)\right\}+ \\
+ & \frac{1}{\tau} \sum_{\mu} p_{\mu}\left\{\left(1-\eta\left(\vec{R}_{c}\right)\right) P\left(\vec{R}_{c}-\vec{e}_{\vec{\mu}}, \eta ; t\right)-\left(1-\eta\left(\vec{R}_{c}+\vec{e}_{\vec{\mu}}\right)\right) P\left(\vec{R}_{c}, \eta ; t\right)\right\}+ \\
+ & \frac{g}{\tau^{*}} \sum_{\vec{r} \neq \vec{R}_{c}}\left\{(1-\eta(\vec{r})) P\left(\vec{R}_{c}, \hat{\eta}^{\vec{r}} ; t\right)-\eta(\vec{r}) P\left(\vec{R}_{c}, \eta ; t\right)\right\}+ \\
+ & \frac{f}{\tau^{*}} \sum_{\vec{r} \neq \vec{R}_{c}}\left\{\eta(\vec{r}) P\left(\vec{R}_{c}, \hat{\eta}^{\vec{r}} ; t\right)-(1-\eta(\vec{r})) P\left(\vec{R}_{c}, \eta ; t\right)\right\} .
\end{aligned}
$$

Note that the terms in the first (resp. second) line of Eq. (3) describe random hopping motion of the "environment" particles (resp. biased motion of the carrier) in terms of the Kawasaki-type particle-vacancy exchanges, while the terms in the third and the fourth lines account for the Glauber-type decay and creation of the "environment" particles.

\subsection{Mean velocity of the charge carrier and correlation functions.}

The velocity of the charge carrier can be now readily determined from Eq.(3). To do it, we multiply both sides of Eq.(3) by $\left(\vec{R}_{c} \cdot \vec{e}_{1}\right)$ and sum over all possible configurations $\left(\vec{R}_{c}, \eta\right)$. This yields the following expression for the carrier mean velocity $V_{c}(t)$ :

$V_{c}(t)=\frac{\sigma}{\tau}\left\{p_{1}\left(1-k\left(\vec{e}_{1} ; t\right)\right)-p_{-1}\left(1-k\left(\vec{e}_{-1} ; t\right)\right)\right\}$

where $k(\vec{\lambda} ; t)$ denotes the carrier-" environment" particles pair correlation function:

$k(\vec{\lambda} ; t) \equiv \sum_{\vec{R}_{c}, \eta} \eta\left(\vec{R}_{c}+\vec{\lambda}\right) P\left(\vec{R}_{c}, \eta ; t\right)$.

Note that $k(\vec{\lambda} ; t)$ is just the density distribution of the "environment" particles, as seen by someone residing on the carrier which moves with velocity $V_{c}(t)$. 
Note now that $V_{c}(t)$ depends explicitly on the local density of the "environment" particles in the immediate vicinity of the carrier. Note also that if the "environment" is perfectly homogeneous, i.e., $k(\vec{\lambda} ; t) \equiv \rho_{s}$, one has that $\eta\left(\vec{R}_{c}+\vec{\lambda}\right)$ and $P\left(\vec{R}_{c}, \eta ; t\right)$ in Eq.(15) decouple, which entails, in virtue of from Eq.(4), a trivial mean-field-type result

$V_{c}^{(0)}=\left(p_{1}-p_{-1}\right)\left(1-\rho_{s}\right) \frac{\sigma}{\tau}$.

The latter (trivial) expression implies that for the perfectly homogeneous, well-stirred environment the frequency of jumps of the carrier particles $\left(\tau^{-1}\right)$ merely gets renormalized by a factor $1-\rho_{s}$, which gives the fraction of successful jumps.

The situation, however, appears to be more complex and there emerge essential backflow effects: As a matter of fact, the carrier effectively perturbs the spatial distribution of the "environment" particles so that stationary density profiles emerge. This can be contrasted to the earlier dynamic percolation models [1415161718202122 23] in which the carrier had no impact on the embedding medium and hence there was no re-arrangement of the host medium around the carrier particle. As a consequence, in our model $k(\vec{\lambda} ; t) \neq \rho_{s}$, and $k(\vec{\lambda} ; t)$ approaches $\rho_{s}$ only at infinite separations from the carrier, i.e. when $|\vec{\lambda}| \rightarrow \infty$. Therefore, we rewrite Eq.(41) in the form

$V_{c}(t)=V_{c}^{(0)}-\frac{\sigma}{\tau}\left\{p_{1}\left(k\left(\vec{e}_{1} ; t\right)-\rho_{s}\right)-p_{-1}\left(\rho_{s}-k\left(\vec{e}_{-1} ; t\right)\right)\right\}$,

which shows explicitly the deviation of the mean velocity of the carrier from the meanfield-type result in Eq.(6) due to the formation of the density profiles.

\subsection{Evolution equations of the pair correlation functions.}

Equation (41) signifies that in order to obtain $V_{c}(t)$ we have to evaluate $k\left(\vec{e}_{ \pm 1} ; t\right)$. Multiplying both sides of Eq. (3) by $\eta\left(\vec{R}_{c}\right)$ and summing over all configurations $\left(\vec{R}_{c}, \eta\right)$, we find that $k(\vec{\lambda} ; t)$ obeys

$$
\begin{aligned}
\partial_{t} k(\vec{\lambda} ; t) & =\frac{l}{6 \tau^{*}} \sum_{\mu}\left(\nabla_{\mu}-\delta_{\vec{\lambda}_{,} \vec{e}_{\mu}} \nabla_{-\mu}\right) k(\vec{\lambda} ; t)-\frac{(f+g)}{\tau^{*}} k(\vec{\lambda} ; t)+\frac{f}{\tau^{*}}+ \\
& +\frac{1}{\tau} \sum_{\mu} \sum_{\vec{R}_{c}, \eta} p_{\mu}\left(1-\eta\left(\vec{R}_{c}+e_{\mu}\right)\right) \nabla_{\mu} \eta\left(\vec{R}_{c}+\vec{\lambda}\right) P\left(\vec{R}_{c}, \eta ; t\right)
\end{aligned}
$$

where $\nabla_{\mu}$ denotes the ascending finite difference operator of the form

$\nabla_{\mu} f(\vec{\lambda}) \equiv f\left(\vec{\lambda}+\vec{e}_{\mu}\right)-f(\vec{\lambda})$

and

$\delta_{\vec{r}, \vec{r}^{\prime}}=\left\{\begin{array}{l}1, \text { if the site } \vec{r}=\vec{r}^{\prime} \\ 0, \text { otherwise. }\end{array}\right.$

The Kroneker-delta term $\delta_{\vec{\lambda}, \vec{e}_{\mu}}$ signifies that the evolution of the pair correlations, Eq.(8), proceeds differently at large separations and in the immediate vicinity of the carrier. This stems from the asymmetric hopping rules of the carrier particle defined by Eq.(2).

Note next that the contribution in the second line in Eq.(8), which stems out of the bias acting on the carrier, is non-linear with respect to the occupation numbers. In 
consequence, the pair correlation function is effectively coupled to the evolution of the third-order correlations. That is, Eq. (8) is not closed with respect to the pair correlations but rather represents a first equation in the infinite hierarchy of coupled equations for higher-order correlation functions. One faces, therefore, the problem of solving an infinite hierarchy of coupled differential equations and needs to resort to an approximate closure scheme.

\subsection{Decoupling Approximation.}

Here we employ a simple non-trivial closure approximation, based on the decoupling of the third-order correlation functions into the product of pair correlations. More precisely, we assume that for $\vec{\lambda} \neq \vec{e}_{\nu}$, the third-order correlation fulfil

$$
\sum_{\vec{R}_{c}, \eta} \eta\left(\vec{R}_{c}+\vec{\lambda}\right) \eta\left(\vec{R}_{c}+\vec{e}_{\nu}\right) P\left(\vec{R}_{c}, \eta ; t\right) \approx k(\vec{\lambda} ; t) k\left(\vec{e}_{\nu} ; t\right)
$$

The approximate closure in Eq.(11) has been already used for studying related models of biased carrier diffusion in hard-core lattice gases and has been shown to provide quite an accurate description of both the dynamical and stationary-state behavior. The decoupling in Eq.(11) was first introduced in Ref. 25] to determine the properties of a driven carrier diffusion in a one-dimensional hard-core lattice gas with a conserved number of particles, i.e. without an exchange of particles with the reservoir. Extensive numerical simulations performed in Ref. 25] have demonstrated that such a decoupling is quite a plausible approximation for the model under study. Moreover, rigorous probabilistic analysis of Ref. 31] has shown that for this model the results based on the decoupling scheme in Eq.(11) are exact. Furthermore, the same closure procedure has been recently applied to study spreading of a hard-core lattice gas from a reservoir attached to one of the lattice sites [32. Again, a very good agreement between the analytical results and the numerical data has been found. Next, the decoupling in Eq.(11) has been used in a recent analysis of a biased carrier dynamics in a one-dimensional model of an adsorbed monolayer in contact with a vapor phase 33, i.e. a one-dimensional version of the model to be studied here. Also in this case an excellent agreement has been observed between the analytical predictions and the Monte Carlo simulations data 33. We now show that the approximate closure of the hierarchy of the evolution equations in Eq.(11) allows us to reproduce in the limit $f, g=0$ and $f / g=$ const the results of Refs. 27] and [28], which are known (see e.g. Ref. 29]) to provide a very good approximation for the carrier diffusion coefficient in three-dimensional hard-core lattice gases with arbitrary particle density. We expect therefore that such a closure scheme will render a plausible description of the carrier dynamics in our three-dimensional generalized dynamic percolation model. We base our further analysis on this approximation.

Making use of Eq.(11), we find from Eq.(8) that the time evolution of pair correlations is governed by the following equations. For all $\vec{\lambda}$ except for $\vec{\lambda}=\left\{\mathbf{0}, \vec{e}_{ \pm 1}, \vec{e}_{ \pm 2}, \vec{e}_{ \pm 3}\right\}$, we have that $k(\vec{\lambda} ; t)$ obeys

$$
\partial_{t} k(\vec{\lambda} ; t)=\frac{l}{6 \tau^{*}} \tilde{L} k(\vec{\lambda} ; t)+\frac{f}{\tau^{*}}
$$


where the operator $\tilde{L}$ and coefficients $A_{\nu}(t)$ are given explicitly by

$\tilde{L} \equiv \sum_{\mu} A_{\mu}(t) \nabla_{\mu}-\frac{6(f+g)}{l}$,

and

$A_{\mu}(t) \equiv 1+\frac{6 \tau^{*}}{l \tau} p_{\mu}\left(1-k\left(\vec{e}_{\mu} ; t\right)\right)$,

the operator $\nabla_{\mu}$ being defined previously in Eq.(9), $\mu=\{ \pm 1, \pm 2, \pm 3\}$. On the other hand, at the sites adjacent to the carrier one has

$\partial_{t} k\left(\vec{e}_{\nu} ; t\right)=\frac{l}{6 \tau^{*}}\left(\tilde{L}+A_{\nu}(t)\right) k\left(\vec{e}_{\nu} ; t\right)+\frac{f}{\tau^{*}}$,

where $\nu=\{ \pm 1, \pm 2, \pm 3\}$.

Now, several comments about equations (12) and (15) are in order. First of all, let us note that Eq.(15) represents, from the mathematical point of view, the boundary conditions for the general evolution equation (12), imposed on the sites in the immediate vicinity of the carrier. Equations (12) and (15) have a different form since in the immediate vicinity of the carrier its asymmetric hopping rules perturb essentially the "environment" particles dynamics. Equations (12) and (15) possess some intrinsic symmetries and hence the number of independent parameters can be reduced. Namely, reversing the field, i.e. changing $E \rightarrow-E$, leads to the mere replacement of $k\left(\vec{e}_{1} ; t\right)$ by $k\left(\vec{e}_{-1} ; t\right)$ but does not affect $k\left(\vec{e}_{\nu} ; t\right)$ with $\nu=\{ \pm 2, \pm 3\}$, which implies that

$k\left(\vec{e}_{1} ; t\right)(-E)=k\left(\vec{e}_{-1} ; t\right)(E)$, and $k\left(\vec{e}_{\nu} ; t\right)(-E)=k\left(\vec{e}_{\nu} ; t\right)(E)$ for $\nu=\{ \pm 2, \pm 3\}$.

Besides, since the transition probabilities in Eq.(2) obey $p_{2}=p_{-2}=p_{3}=p_{-3}$, one evidently has that $k\left(\vec{e}_{2} ; t\right)=k\left(\vec{e}_{-2} ; t\right)=k\left(\vec{e}_{3} ; t\right)=k\left(\vec{e}_{-3} ; t\right)$, and hence, by symmetry, $A_{2}(t)=A_{-2}(t)=A_{3}(t)=A_{-3}(t)$, which somewhat simplifies equations (12) and (15). Lastly, we note that despite the fact that using the decoupling scheme in Eq.(11) we effectively close the system of equations on the level of the pair correlations, the solution of Eqs.(12) and (15) still poses serious technical difficulties. Namely, these equations are strongly non-linear with respect to the carrier velocity, which introduces the gradient term on the rhs of the evolution equations for the pair correlation, and depends by itself on the values of the "environment" particles densities in the immediate vicinity of the carrier. Below we discuss a solution to this non-linear problem, focusing on the limit $t \rightarrow \infty$.

\section{SOLUTION OF THE DECOUPLED EVOLUTION EQUATIONS IN THE STATIONARY STATE.}

Consider the limit $t \rightarrow \infty$ and suppose that the density profiles and the stationary velocity of the carrier have non-trivial stationary values: $k(\vec{\lambda}) \equiv \lim _{t \rightarrow \infty} k(\vec{\lambda} ; t), V_{c} \equiv$ $\lim _{t \rightarrow \infty} V_{c}(t)$ and $A_{\mu}=\lim _{t \rightarrow \infty} A_{\mu}(t)$. As the next step, we define the local deviations of $k(\vec{\lambda})$ from the unperturbed density: $h(\vec{\lambda}) \equiv k(\vec{\lambda})-\rho_{s}$. This yields the following system of equations:

$\tilde{L} h(\vec{\lambda})=0$, 
for $\vec{\lambda} \neq\left\{\mathbf{0}, \vec{e}_{ \pm 1}, \vec{e}_{ \pm 2}, \vec{e}_{ \pm 3}\right\}$, while for the special sites adjacent to the carrier one has $\left(\tilde{L}+A_{\nu}\right) h\left(\vec{e}_{\nu}\right)+\rho_{s}\left(A_{\nu}-A_{-\nu}\right)=0$,

Equations (17) and (18) determine the spatial distribution of the deviation from the unperturbed density $\rho_{s}$ in the stationary state. Note also that in virtue of the symmetry relations $h\left(\vec{e}_{ \pm 2}\right)=h\left(\vec{e}_{ \pm 3}\right)$ and $A_{2}=A_{-2}=A_{3}=A_{-3}$.

To solve the coupled non-linear Eqs.(44),(17) and (18) we proceed in the following, standard manner: We first solve these equations supposing that the carrier stationary velocity is a fixed, given parameter (and hence, the functions $A_{\nu}$ entering Eqs.(17) and (18) are known). In doing so, we obtain $h(\lambda)$ in the parameterized form $h(\vec{\lambda})=h\left(\vec{\lambda} ; A_{ \pm 1}, A_{2}\right)$. Then, choosing particular values $\vec{\lambda}=\left\{\vec{e}_{ \pm 1}, \vec{e}_{ \pm 2}, \vec{e}_{ \pm 3}\right\}$ and making use of the definition of $A_{\mu}$, we find a system of three linear equations with three unknowns of the form

$A_{\nu}=1+\frac{6 \tau^{*}}{l \tau} p_{\nu}\left(1-\rho_{s}-h\left(\vec{e}_{\nu} ; A_{ \pm 1}, A_{2}\right)\right)$,

where $\nu=\{ \pm 1,2\}$, which will allow us to obtain a closure relation and hence, to define all $A_{\nu}$ explicitly (and hence, all $h\left(\vec{e}_{\nu}\right)$ ). Finally, substituting the results into Eq.(44), which can be written down in terms of $A_{\nu}$ as

$V_{c}=\frac{l \sigma}{6 \tau^{*}}\left(A_{1}-A_{-1}\right)$,

we arrive at a closed-form equation determining implicitly the stationary velocity.

\subsection{Density profiles in the dynamic percolative environment.}

The general solution of Eqs.(17) and (18) can be obtained in a standard fashion by introducing the following generating function:

$H\left(w_{1}, w_{2}, w_{3}\right) \equiv \sum_{n_{1}, n_{2}, n_{3}} h(\vec{\lambda}) w_{1}^{n_{1}} w_{2}^{n_{2}} w_{3}^{n_{3}}$,

where $n_{1}, n_{2}$ and $n_{3}$ are the components of the vector $\vec{\lambda}, \vec{\lambda}=\vec{e}_{1} n_{1}+\vec{e}_{2} n_{2}+\vec{e}_{3} n_{3}$. Multiplying both sides of Eqs. (17) and (18) by $w_{1}^{n_{1}} w_{2}^{n_{2}} w_{3}^{n_{3}}$ and performing summation, we find then that $H\left(w_{1}, w_{2}, w_{3}\right)$ is given explicitly by

$H\left(w_{1}, w_{2}, w_{3}\right)=-l \frac{\sum_{\nu}\left(A_{\nu}\left(w_{|\nu|}^{\nu /|\nu|}-1\right) h\left(\vec{e}_{\nu}\right)+\rho_{s}\left(A_{\nu}-A_{-\nu}\right) w_{|\nu|}^{\nu}\right)}{l \sum_{\nu} A_{\nu}\left(w_{|\nu|}^{-\nu /|\nu|}-1\right)-6(f+g)}$,

an expression which allows us to determine the stationary density profiles as seen from the carrier which moves with a constant velocity $V_{c}$.

Inverting next the generating function, Eq.(22), we get, after rather lengthy but straightforward calculations, the following explicit result for the local deviation from the unperturbed density:

$h(\vec{\lambda})=\alpha^{-1}\left\{\sum_{\nu} A_{\nu} h\left(\vec{e}_{\nu}\right) \nabla_{-\nu}-\rho_{s}\left(A_{1}-A_{-1}\right)\left(\nabla_{1}-\nabla_{-1}\right)\right\} F(\vec{\lambda})$,

where $F(\vec{\lambda})$ is given by

$F(\vec{\lambda})=\left(\frac{A_{-1}}{A_{1}}\right)^{n_{1} / 2} \int_{0}^{\infty} e^{-x} \mathrm{I}_{n_{1}}\left(2 \frac{\sqrt{A_{1} A_{-1}}}{\alpha} x\right) \mathrm{I}_{n_{2}}\left(2 \frac{A_{2}}{\alpha} x\right) \mathrm{I}_{n_{3}}\left(2 \frac{A_{2}}{\alpha} x\right) \mathrm{d} x$, 
and

$\alpha=\sum_{\nu} A_{\nu}+\frac{6(f+g)}{l}=A_{1}+A_{-1}+4 A_{2}+\frac{6(f+g)}{l}$

Consequently, the particles density distribution as seen from the carrier moving with a constant velocity $V_{c}$ obeys

$k(\vec{\lambda})=\rho_{s}+\alpha^{-1}\left\{\sum_{\nu} A_{\nu} h\left(\vec{e}_{\nu} \nabla_{-\nu}-\rho_{s}\left(A_{1}-A_{-1}\right)\left(\nabla_{1}-\nabla_{-1}\right)\right\} F(\vec{\lambda})\right.$,

where we have to determine three yet unknown parameters $A_{1}, A_{-1}$ and $A_{2}$.

To determine these parameters, we set in Eq.(23) $\vec{\lambda}=\vec{e}_{1}, \vec{\lambda}=\vec{e}_{-1}$ and $\vec{\lambda}=\vec{e}_{2}$, which results in the system of three closed-form equations determining the unknown functions $A_{\nu}, \nu=\{ \pm 1,2\}$,

$A_{\nu}=1+\frac{6 \tau^{*}}{l \tau} p_{\nu}\left\{1-\rho_{s}-\rho_{s}\left(A_{1}-A_{-1}\right) \frac{\operatorname{det} \tilde{C}_{\nu}}{\operatorname{det} \tilde{C}}\right\}$

where $\tilde{C}$ is a square matrix of the third order defined as

$$
\left(\begin{array}{ccc}
A_{1} \nabla_{-1} F\left(\vec{e}_{1}\right)-\alpha & A_{-1} \nabla_{1} F\left(\vec{e}_{1}\right) & A_{2} \nabla_{-2} F\left(\vec{e}_{1}\right) \\
A_{1} \nabla_{-1} F\left(\vec{e}_{-1}\right) & A_{-1} \nabla_{1} F\left(\vec{e}_{-1}\right)-\alpha & A_{2} \nabla_{-2} F\left(\vec{e}_{-1}\right) \\
A_{1} \nabla_{-1} F\left(\vec{e}_{2}\right) & A_{-1} \nabla_{1} F\left(\vec{e}_{2}\right) & A_{2} \nabla_{-2} F\left(\vec{e}_{2}\right)-\alpha
\end{array}\right)
$$

while $\tilde{C}_{\nu}$ stands for the matrix obtained from $\tilde{C}$ by replacing the $\nu$-th column by a column vector $\left(\left(\nabla_{1}-\nabla_{-1}\right) F\left(\vec{e}_{\nu}\right)\right)_{\nu}$. Equation (26) , together with the definition of the coefficients $A_{\nu}$, constitutes the first general result of our analysis defining the density distribution in the percolative environment under study.

\subsection{General force-velocity relation.}

Substituting Eq.(27) into (20), we find that the stationary velocity of the carrier particle is defined implicitly as the solution of equation:

$V_{c}=\frac{\sigma}{\tau}\left(p_{1}-p_{-1}\right)\left(1-\rho_{s}\right)\left\{1+\rho_{s} \frac{6 \tau^{*}}{l \tau} \frac{p_{1} \operatorname{det} \tilde{C}_{1}-p_{-1} \operatorname{det} \tilde{C}_{-1}}{\operatorname{det} \tilde{C}}\right\}^{-1}$,

where $\tilde{C}_{1}$ and $\tilde{C}_{-1}$ are the following square matrices of the third order:

$\tilde{C}_{1}=\left(\begin{array}{ccc}\left(\nabla_{1}-\nabla_{-1}\right) F\left(\vec{e}_{1}\right) & A_{-1} \nabla_{1} F\left(\vec{e}_{1}\right) & A_{2} \nabla_{-2} F\left(\vec{e}_{1}\right) \\ \left(\nabla_{1}-\nabla_{-1}\right) F\left(\vec{e}_{-1}\right) & A_{-1} \nabla_{1} F\left(\vec{e}_{-1}\right)-\alpha & A_{2} \nabla_{-2} F\left(\vec{e}_{-1}\right) \\ \left(\nabla_{1}-\nabla_{-1}\right) F\left(\vec{e}_{2}\right) & A_{-1} \nabla_{1} F\left(\vec{e}_{2}\right) & A_{2} \nabla_{-2} F\left(\vec{e}_{2}\right)-\alpha\end{array}\right)$

and

$\tilde{C}_{-1}=\left(\begin{array}{ccc}A_{1} \nabla_{-1} F\left(\vec{e}_{1}\right)-\alpha & \left(\nabla_{1}-\nabla_{-1}\right) F\left(\vec{e}_{1}\right) & A_{2} \nabla_{-2} F\left(\vec{e}_{1}\right) \\ A_{1} \nabla_{-1} F\left(\vec{e}_{-1}\right) & \left(\nabla_{1}-\nabla_{-1}\right) F\left(\vec{e}_{-1}\right) & A_{2} \nabla_{-2} F\left(\vec{e}_{-1}\right) \\ A_{1} \nabla_{-1} F\left(\vec{e}_{2}\right) & \left(\nabla_{1}-\nabla_{-1}\right) F\left(\vec{e}_{2}\right) & A_{2} \nabla_{-2} F\left(\vec{e}_{2}\right)-\alpha\end{array}\right)$.

Equation (29) represents our second principal result defining the force-velocity relation in the dynamic percolative environment for an arbitrary field and arbitrary rates of the diffusive and renewal processes. 


\section{CARRIER VELOCITY, FRICTION AND DIFFUSION COEFFICIENTS.}

Consider now the case when the applied external field $E$ is small. Expanding the transition probabilities $p_{1}$ and $p_{-1}$ in the Taylor series up to the first order in powers of the external field, i.e., setting

$p_{ \pm 1}=\frac{1}{6} \pm \frac{\sigma \beta E}{12}+\mathcal{O}\left(E^{2}\right)$,

we find that $V_{c}$, Eq.(20), follows

$V_{c} \sim \frac{\sigma}{6 \tau}\left\{\sigma \beta E\left(1-\rho_{s}\right)-\left(h\left(\vec{e}_{1}\right)-h\left(\vec{e}_{-1}\right)\right)\right\}$.

On the other hand, Eq.(23) entails that

$h\left(\vec{e}_{1}\right)-h\left(\vec{e}_{-1}\right)=\frac{2 \sigma \rho_{s}\left(1-\rho_{s}\right) \tau^{*}}{l \tau\left(\alpha_{0} \mathcal{L}\left(2 A_{0} / \alpha_{0}\right)-A_{0}\right)+2 \rho_{s} \tau} \beta E+\mathcal{O}\left(E^{2}\right)$,

where

$A_{0}=\lim _{E \rightarrow 0} A_{\nu}=1+\frac{\tau^{*}}{l \tau}\left(1-\rho_{s}\right)$,

and

$\alpha_{0}=\lim _{E \rightarrow 0} \alpha=6\left(1+\frac{\tau^{*}\left(1-\rho_{s}\right)}{l \tau}+\frac{f+g}{l}\right)$,

while

$\mathcal{L}(x) \equiv\left\{\int_{0}^{\infty} e^{-t} \mathrm{I}_{0}^{2}(x t)\left(\mathrm{I}_{0}(x t)-\mathrm{I}_{2}(x t)\right) \mathrm{d} t\right\}^{-1}=\left\{P(\mathbf{0} ; 3 x)-P\left(2 \vec{e}_{1} ; 3 x\right)\right\}^{-1}$,

with $P(\vec{r} ; \xi)$ being the generating function,

$P(\vec{r} ; \xi) \equiv \sum_{j=0}^{+\infty} P_{j}(\vec{r}) \xi^{j}$,

of the probability $P_{j}(\vec{r})$ that a walker starting at the origin and performing a Polya random walk on the sites of a three-dimensional cubic lattice will arrive on the $j$-th step to the site with the lattice vector $\vec{r}[4$.

Consequently, we find that in the limit of a small applied field $E$ the force-velocity relation in Eq.(29) attains the physically meaningful form of the Stokes formula $E=\zeta V_{c}$, which signifies that the frictional force exerted on the carrier by the environment particles is viscous. The effective friction coefficient $\zeta$ is the sum of two terms,

$\zeta=\zeta_{0}+\zeta_{\text {coop }}$

where the first term represents a mean-field-type result $\zeta_{0}=6 \tau / \beta \sigma^{2}\left(1-\rho_{s}\right)$ (see Eq.(7)), while the second one, $\zeta_{c o o p}$, obeys

$\zeta_{\text {coop }}=\frac{12 \rho_{s} \tau^{*}}{\beta \sigma^{2} l\left(1-\rho_{s}\right) \cdot\left(\alpha_{0} \mathcal{L}\left(2 A_{0} / \alpha_{0}\right)-A_{0}\right)}$ 
The second contribution has a more complicated origin and is associated with the cooperative behavior - formation of a inhomogeneous stationary particle distribution around the carrier moving with constant velocity $V_{c}$. Needless to say, such an effect can not be observed within the framework of previous models of dynamic percolation, since there the carrier does not influence the host medium dynamics 1415161718202122 23.

Let us now compare the relative importance of two contributions, i.e. $\zeta_{0}$ and $\zeta_{\text {coop }}$, to the overall friction. Straightforward analysis shows that the cooperative behavior dominates at small and moderate $f$ (which entails also small values of $g$ ), while for larger $f$, when $\zeta / \zeta_{0}$ tends to 1 , the mean-field behavior becomes most important. The cooperative behavior also appears to be more pronounced at larger densities $\rho_{s}$.

Consider next some analytical estimates. We start with the situation, in which diffusion of the environment particles is suppressed, i.e. when $l=0$. In this case, we get

$\frac{\zeta_{\text {coop }}}{\zeta_{0}}=\frac{2 \rho_{s}}{\left(1-\rho_{s}\right)\left(\frac{2}{y} \mathcal{L}(y)-1\right)}$,

where

$y=\frac{1}{3}\left(1+\frac{\tau}{\tau^{*}} \frac{(f+g)}{\left(1-\rho_{s}\right)}\right)^{-1}$.

Suppose first that $\rho_{s}$ is small, $\rho_{s} \ll 1$. Then, $y \approx 1 / 3\left(1+\tau / \tau^{*}(f+g)\right)$ and we can distinguish between two situations: when $\tau \ll(f+g) / \tau^{*}$, i.e. when the carrier moves faster than the environment re-organizes itself, and the opposite limit, $\tau \gg(f+g) / \tau^{*}$, when the environment changes very rapidly compared to the motion of the carrier. In the former case we find that $y \approx 1 / 3$, which yields $\zeta_{\text {coop }} / \zeta_{0} \approx 2 \rho_{s} /(6 \mathcal{L}(1 / 3)-1), \mathcal{L}(1 / 3) \approx 0.7942$, while in the latter case we have $y \approx \tau^{*} / 3 \tau(f+g)$ and $\zeta_{\text {coop }} / \zeta_{0} \approx \rho_{s} \tau^{*} / 3 \tau(f+g)$. Note, that in both cases the ratio $\zeta_{\text {coop }} / \zeta_{0}$ appears to be small, which signifies that at small densities $\rho_{s}$ the mean-field friction dominates. Such a result is not counterintuitive, of course, since in the absence of the particles' diffusion, which couples effectively the density evolution at different lattice sites, no significant cooperative behavior can emerge at small densities. On the other hand, at relatively high densities $\rho_{s} \sim 1$ and $\tau /\left(1-\rho_{s}\right) \gg \tau^{*} /(f+g) \gg \tau$, when the carrier moves at much faster rate than the host medium reorganizes itself, we find that $\zeta_{\text {coop }} / \zeta_{0} \approx \tau^{*} / 3 \tau(f+g) \gg 1$. This result stems from the circumstance that in sufficiently dense environments modeled by dynamic percolation a highly inhomogeneous density profile emerges even in the absence of particles diffusion. Here, on the one hand, the carrier perturbs significantly the particle density in its immediate vicinity. On the other hand, the density perturbation created by the carrier does not shift the global balance between creation and annihilation events, i.e. the mean particle density still equals $\rho_{s}$. The latter constraint induces then appearance of essential correlations in particles distribution and hence, appearance of cooperative behavior.

Let us consider the opposite case when the renewal processes are not allowed, which means that the particles number is conserved and local density in the percolative environment evolves only due to particles diffusion. In this case we find

$\frac{\zeta_{\text {coop }}}{\zeta_{0}}=\frac{2 \tau^{*} \rho_{s}}{\left(l \tau+\tau^{*}\left(1-\rho_{s}\right)\right)(6 \mathcal{L}(1 / 3)-1)}$ 
Here, the ratio $\zeta_{\text {coop }} / \zeta_{0}$ can be large and the "cooperative" friction dominates the meanfield one when $l \tau \ll \tau^{*}\left(3 \rho_{s}-1\right)$, which happens, namely, at sufficiently high densities and in the limit when the carrier moves at a much faster rate than the environment reorganizes itself. Otherwise, the mean-field friction prevails.

To estimate the carrier particle diffusion coefficient $D_{c}$ we assume the validity of the Einstein relation, i.e. $\beta D_{c}=\zeta^{-1}$ (see, e.g., Ref.[26]). We find that, in the general case, the carrier diffusion coefficient $D_{c}$ reads

$D_{c}=\frac{\sigma^{2}\left(1-\rho_{s}\right)}{6 \tau}\left\{1-\frac{2 \rho_{s} \tau^{*}}{l \tau}\left(\alpha_{0} \mathcal{L}\left(2 A_{0} / \alpha_{0}\right)-1+\frac{\tau^{*}\left(3 \rho_{s}-1\right)}{l \tau}\right)^{-1}\right\}$

In the particular case of conserved particles number, when $f, g \rightarrow 0$ but their ratio $f / g$ is kept fixed, $f / g=\rho_{s} /\left(1-\rho_{s}\right)$, the latter equation reduces to the classical result

$D_{c}^{N K}=\frac{\sigma^{2}\left(1-\rho_{s}\right)}{6 \tau}\left\{1-\frac{2 \rho_{s} \tau^{*}}{l \tau}\left(6 A_{0} \mathcal{L}(1 / 3)-1+\frac{\tau^{*}\left(3 \rho_{s}-1\right)}{l \tau}\right)^{-1}\right\}$,

obtained earlier in Refs. 27] and [28] by different analytical techniques. The result in Eq. (45) is known to be exact in the limits $\rho_{s} \ll 1$ and $\rho_{s} \sim 1$, and serves as a very good approximation for the self-diffusion coefficient in hard-core lattice gases of arbitrary density [29].

Finally, in the absence of particle diffusion (fluctuating-site percolation), our result for the carrier particle diffusion coefficient reduces to

$D_{c}^{\text {per }}=\frac{\sigma^{2}\left(1-\rho_{s}\right)}{6 \tau}\left\{1-2 \rho_{s}\left(4\left[\left(1-\rho_{s}\right)+(f+g) \tau / \tau^{*}\right] \mathcal{L}(y)+3 \rho_{s}-1\right)^{-1}\right\}$

Note, however, that this result only applies when both $f$ and $g$ are larger than zero, such that the renewal processes take place. In fact, the underlying decoupling scheme is only plausible in this case. Similarly to the approximate theories in Refs. 27] and [28, our approach predicts that in the absence of the renewal processes $D_{c}^{\text {per }}$ vanishes only when $\rho_{s} \rightarrow 1$, which is an incorrect behavior.

\section{ASYMPTOTIC BEHAVIOR OF THE DENSITY PROFILES.}

The density profiles at large separations in front of and past the carrier can be readily deduced from the asymptotical behavior of the following generating function

$N\left(w_{1}\right) \equiv \sum_{n_{1}=-\infty}^{+\infty} h\left(n_{1}, n_{2}=0, n_{3}=0\right) w_{1}^{n_{1}}$

Inversion of Eq.(22) with respect to the symmetric coordinates $n_{2}$ and $n_{3}$ yields then

$$
\begin{aligned}
& N\left(w_{1}\right)=\frac{\left(A_{1} h\left(\vec{e}_{1}\right)+\rho_{s}\left(A_{1}-A_{-1}\right)\right)\left(w_{1}-1\right)+\left(A_{-1} h\left(\vec{e}_{-1}\right)-\rho_{s}\left(A_{1}-A_{-1}\right)\right)\left(w_{1}^{-1}-1\right)}{\alpha-A_{1} w_{1}^{-1}-A_{-1} w_{1}} \times \\
\times & \int_{0}^{\infty} \exp [-x] \mathrm{I}_{0}^{2}\left(\frac{2 A_{2}}{\alpha-A_{1} w_{1}^{-1}-A_{-1} w_{1}} x\right) \mathrm{d} x+\frac{4 A_{2} h\left(\vec{e}_{2}\right)}{\alpha-A_{1} w_{1}^{-1}-A_{-1} w_{1}} \times \\
\times & \int_{0}^{\infty} \exp [-x] \mathrm{I}_{0}\left(\frac{2 A_{2}}{\alpha-A_{1} w_{1}^{-1}-A_{-1} w_{1}} x\right)\left(\mathrm{I}_{1}\left(\frac{2 A_{2}}{\alpha-A_{1} w_{1}^{-1}-A_{-1} w_{1}} x\right)-\right. \\
- & \left.\mathrm{I}_{0}\left(\frac{2 A_{2}}{\alpha-A_{1} w_{1}^{-1}-A_{-1} w_{1}} x\right)\right) \mathrm{d} x
\end{aligned}
$$


We notice now that $N\left(w_{1}\right)$ is a holomorphic function in the region $\mathcal{W}_{1}<w_{1}<\mathcal{W}_{2}$, where

$\mathcal{W}_{1}=\frac{\alpha-4 A_{2}}{2 A_{-1}}-\sqrt{\left(\frac{\alpha-4 A_{2}}{2 A_{-1}}\right)^{2}-\frac{A_{1}}{A_{-1}}}$

and

$\mathcal{W}_{2}=\frac{\alpha-4 A_{2}}{2 A_{-1}}+\sqrt{\left(\frac{\alpha-4 A_{2}}{2 A_{-1}}\right)^{2}-\frac{A_{1}}{A_{-1}}}$

As a consequence, the asymptotic behavior of $h\left(n_{1}, n_{2}=0, n_{3}=0\right)$ in the limit $n_{1} \rightarrow \infty$ (resp. $n_{1} \rightarrow-\infty$ ) is controlled by the behavior of $N\left(w_{1}\right)$ in the vicinity of $w_{1}=\mathcal{W}_{2}$ (resp. $w_{1}=\mathcal{W}_{1}$ ) (see, for example, the analysis of the generating function singularities developed in Ref. 34]).

\subsection{Asymptotics of the density profiles at large separations in front of the carrier.}

Consider first the asymptotic behavior of the density distribution of the "environment" particles at large separations in front of the carrier. We find then that in the limit $w_{1} \rightarrow \mathcal{W}_{2}$, the function $N\left(w_{1}\right)$ follows

$$
\begin{aligned}
N\left(w_{1}\right) & \sim{ }_{w_{1} \rightarrow \mathcal{W}_{2}}\left[\frac{\left(A_{1} h\left(\vec{e}_{1}\right)+\rho_{s}\left(A_{1}-A_{-1}\right)\right)\left(\mathcal{W}_{2}-1\right)}{4 \pi A_{2}}+\right. \\
& \left.+\frac{\left(A_{-1} h\left(\vec{e}_{-1}\right)-\rho_{s}\left(A_{1}-A_{-1}\right)\right)\left(\mathcal{W}_{2}^{-1}-1\right)}{4 \pi A_{2}}\right] \ln \left(\mathcal{W}_{2}-w_{1}\right)
\end{aligned}
$$

Then, (cf, Ref. [34]), we obtain the following asymptotical result

$h\left(n_{1}, 0,0\right) \sim_{n_{1} \rightarrow \infty} \frac{K^{+}}{n_{1}} e^{-n_{1} / \lambda_{+}}$,

where the characteristic length $\lambda_{+}$is given explicitly by

$\lambda_{+} \equiv \ln ^{-1}\left(\frac{\alpha / 2-2 A_{2}}{A_{-1}}+\sqrt{\left(\frac{\alpha / 2-2 A_{2}}{A_{-1}}\right)^{2}-\frac{A_{1}}{A_{-1}}}\right)$,

and the amplitude $K^{+}$obeys

$$
\begin{aligned}
K^{+} & =\left[\frac{\left(A_{1} h\left(\vec{e}_{1}\right)+\rho_{s}\left(A_{1}-A_{-1}\right)\right)\left(\mathcal{W}_{2}-1\right)}{4 \pi A_{2}}+\right. \\
& \left.+\frac{\left(A_{-1} h\left(\vec{e}_{-1}\right)-\rho_{s}\left(A_{1}-A_{-1}\right)\left(\mathcal{W}_{2}^{-1}-1\right)\right.}{4 \pi A_{2}}\right]>0,
\end{aligned}
$$

which signifies that the density of the "environment" particles in front of the carrier is higher than the average value $\rho_{s}$ and approaches $\rho_{s}$ at large separations from the carrier as an exponential function of the distance. 


\subsection{Asymptotics of the density profiles at large separations behind the carrier.} We consider next the asymptotic behavior of the "environment" particles density profiles past the carrier particle, which turns out to be very different depending on whether the dynamics of the percolative environment obeys the strict conservation of the "environment" particles number or not (the renewal processes are suppressed or allowed).

\subsubsection{Non-conserved particles number.}

In the case when particles may disappear and re-appear on the lattice, one has that the root $\mathcal{W}_{1}<1$. We find then, following essentially the same lines as in the previous subsection, that

$$
\begin{aligned}
N\left(w_{1}\right) & \sim{ }_{w_{1} \rightarrow \mathcal{W}_{1}}\left[\frac{\left(A_{1} h\left(\vec{e}_{1}\right)+\rho_{s}\left(A_{1}-A_{-1}\right)\right)\left(\mathcal{W}_{1}-1\right)}{4 \pi A_{2}}+\right. \\
& \left.+\frac{\left(A_{-1} h\left(\vec{e}_{-1}\right)-\rho_{s}\left(A_{1}-A_{-1}\right)\right)\left(\mathcal{W}_{1}^{-1}-1\right)}{4 \pi A_{2}}\right] \ln \left(\frac{1}{w_{1}-\mathcal{W}_{1}}\right) .
\end{aligned}
$$

Hence, in the non-conserved case the approach to the unperturbed value $\rho_{s}$ is also exponential when $n_{1} \rightarrow-\infty$, and follows

$h_{n_{1}, 0,0} \sim_{n_{1} \rightarrow-\infty} \frac{K^{-}}{\left|n_{1}\right|} e^{-\left|n_{1}\right| / \lambda_{-}}$,

where

$$
\lambda_{-} \equiv \ln ^{-1}\left(\frac{\alpha / 2-2 A_{2}}{A_{-1}}-\sqrt{\left(\frac{\alpha / 2-2 A_{2}}{A_{-1}}\right)^{2}-\frac{A_{1}}{A_{-1}}}\right)
$$

and

$$
\begin{aligned}
K^{-} & =\left[\frac{\left(A_{1} h\left(\vec{e}_{1}\right)+\rho_{s}\left(A_{1}-A_{-1}\right)\right)\left(\mathcal{W}_{1}-1\right)}{4 \pi A_{2}}+\right. \\
& \left.+\frac{\left(A_{-1} h\left(\vec{e}_{-1}\right)-\rho_{s}\left(A_{1}-A_{-1}\right)\right)\left(\mathcal{W}_{1}^{-1}-1\right)}{4 \pi A_{2}}\right]<0
\end{aligned}
$$

which implies that the particles density past the carrier is lower than the average. Note that, in the general case, $\lambda_{+}<\lambda_{-}$, which means that the depleted region past the carrier is more extended in space than the traffic-jam-like region in front of the carrier. The density profiles are therefore asymmetric with respect to the origin, $n_{1}=0$.

\subsubsection{Conserved particles number.}

Finally, we turn to the analysis of the shape of the density profiles of the percolative environment behind the carrier in the particular limit when the host medium evolves only due to diffusion, while creation and annihilation of particles are completely suppressed. In this case, in which the particles number is explicitly conserved, one has that for arbitrary value of the field and particles' average density, the root $\mathcal{W}_{1} \equiv 1$ and, consequently, the form of the generating function is qualitatively different from that in Eqs.(51) and (55),

$$
N\left(w_{1}\right) \sim_{w_{1} \rightarrow 1^{+}}\left[\frac{\left(A_{1} h\left(\vec{e}_{1}\right)-A_{-1} h\left(\vec{e}_{-1}\right)\right.}{4 \pi A_{2}}+\frac{\left.2 \rho_{s}\left(A_{1}-A_{-1}\right)\right)}{4 \pi A_{2}}\right]\left(w_{1}-1\right) \ln \left(\frac{1}{w_{1}-1}\right) .
$$


Equation (59) implies that in the limit when the particle number is conserved the large- $n_{1}$ asymptotic behavior of $h_{n_{1}, 0,0}$ is described by an algebraic function of $n_{1}$ with a logarithmic correction; that is,

$h_{n_{1}, 0,0} \sim \frac{K_{-} \ln \left(\left|n_{1}\right|\right)}{n_{1}^{2}}$,

where $K_{-}$is an $n_{1}$-independent constant. Remarkably, the power-law decay of correlations implies existence of a quasi-long-range order in the percolative environment past the carrier. In the conserved case the mixing of the three-dimensional percolative environment is not very efficient and there are considerable memory effects - the host medium remembers the passage of the carrier on large space and time scales.

\section{CONCLUSIONS}

To conclude, we have presented a microscopic model describing the dynamics of a charge carrier, driven by an external field $\vec{E}$ in a three-dimensional complex medium modeled by dynamic percolation, i. e. represented as a cubic lattice partially filled with mobile, hardcore "environment" particles which can spontaneously disappear and reappear (renewal processes) in the system with some prescribed rates. Our analytical description of the transfer process in such a medium has been based on the master equation, describing the time evolution of the system, which has allowed us to evaluate a system of coupled dynamical equations for the charge carrier velocity and a hierarchy of correlation functions. To solve these coupled equations, we have invoked an approximate closure scheme based on the decomposition of the third-order correlation functions into a product of pairwise correlations, which has been first introduced in Ref. 25] for a related model of a driven carrier dynamics in a one-dimensional lattice gas with conserved particles number. Within the framework of this approximation, we have derived a system of coupled, discrete-space equations describing evolution of the density profiles of the environment, as seen from the moving charge carrier, and its velocity $V_{c}$. We have shown that $V_{c}$ depends on the density of the "environment" particles in front of and past the carrier. Both densities depend on the magnitude of the velocity, as well as on the rate of the renewal and diffusive processes. As a consequence of such a non-linear coupling, in the general case, (i.e. for an arbitrary driving field and arbitrary rates of renewal and diffusive processes), $V_{c}$ has been found only implicitly, as the solution of a non-linear equation relating its value to the system parameters. This equation, which defines the force-velocity relation for the dynamic percolation under study, simplifies considerably in the limit of small applied field $\vec{E}$. We find that in this limit it attains the physically meaningful form of the Stokes formula, which implies, in particular, that the frictional force exerted on the carrier by the environment modeled by dynamic percolation is viscous. In this limit, the carrier velocity and the friction coefficient are calculated explicitly. In addition, we determined the selfdiffusion coefficient of the carrier in the absence of the field and show that it reduces to the well-know result of Refs. 27] and [28] in the limit when the particles number is conserved. Further more, we have found that the density profile around the carrier becomes strongly inhomogeneous: the local density of the "environment" particles in front of the carrier is higher than the average and approaches the average value as an exponential function of the distance from the carrier. On the other hand, behind the carrier the local density is 
lower than the average, and depending on whether the number of particles is explicitly conserved or not, the local density past the carrier may tend to the average value either as an exponential or even as an algebraic function of the distance. The latter reveals especially strong memory effects and strong correlations between the particle distribution in the environment and the carrier position.

\section{Acknowledgments.}

G.O. acknowledges the financial support from the Alexander von Humboldt Foundation via the Bessel Research Award.

\section{REFERENCES}

1. A.M.Kuznetsov, Charge Transfer in Physics, Chemistry, and Biology, Gordon and Breach, Reading, 1995

2. S.Havlin and D.Ben-Avraham, Advances in Physics 36 (1987) 695

3. M.Sahimi, Applications of Percolation Theory, Taylor and Francis, London, 1994

4. B.D.Hughes, Random Walks and Random Environments, Clarendon Press, Oxford, 1996

5. E.E.Polymeropoulos and J.Brickmann, Ann. Rev. Biophys. 14 (1985) 315

6. S.Chandra, Superionic Solids, Principles and Applications, North-Holland, Amsterdam, 1981

7. S.Bhattarcharya, J.P.Stokes, M.W.Kim, and J.S.Huang, Phys. Rev. Lett. 55 (1985) 1884

8. G.S.Grest, I.Webman, S.A.Safran, and A.L.R.Bug, Phys. Rev. A 33 (1986) 2842

9. A.L.R.Bug and Y.Gefan, Phys. Rev. A 35 (1987) 1301

10. C.M.C.Gambi, M.G.Giri, M.Carlà, D.Senatra, and A.Chittofrati, Phys. Rev. E 56 (1997) 4356

11. H.Cheradame, J.L.Souquet and J.M.Latour, Mater. Res. Bull. 15 (1980) 1173

12. B.L.Papke, R.Dupon, M.A.Ratner and D.F.Shriver, Solid State Ion. 5 (1981) 685

13. C.A.Vincent, Prog. Solid State Chem. 17 (1987) 145

14. S.D.Druger, A.Nitzan, and M.A.Ratner, J. Chem. Phys. 79 (1983) 3133

15. S.D.Druger, M.A.Ratner, and A.Nitzan, Phys. Rev. B 31 (1985) 3939

16. A.K.Harrison and R.Zwanzig, Phys. Rev. A 32 (1985) 1072

17. M.Sahimi, J. Phys. C 19 (1986) 1311

18. A.P.Chatterjee and R.F.Loring, Phys. Rev. E 50 (1994) 2439

19. T.Odagaki and M.Lax, Phys. Rev. B 24 (1981) 5284

20. R.Granek and A.Nitzan, J. Chem. Phys. 90 (1989) 3784

21. E.Hernández-Garcia, L.Pesquera, M.A.Rodriguez, and M.San Miguel, J. Stat. Phys. 55 (1989) 1027

22. R.Hilfer and R.Orbach, Chem. Phys. 128 (1988) 275

23. R.Granek and A.Nitzan, J. Chem. Phys. 92 (1990) 1329

24. O.Bénichou, J.Klafter, M.Moreau, and G.Oshanin, Phys. Rev. E 62 (2000) 3327

25. S.F.Burlatsky, G.Oshanin, A.Mogutov and M.Moreau, Phys. Lett. A 166 (1992) 230; S.F.Burlatsky, G.Oshanin, M.Moreau and W.P.Reinhardt, Phys. Rev. E 54 (1996) 3165 
26. G.Oshanin, O.Bénichou, M.Moreau, and S.F.Burlatsky, Biased Tracer Diffusion in Hard-Core Lattice Gases: Some Notes on the Validity of the Einstein Relation, in: Instabilities and Non-Equilibrium Structures IX, ed.: O.Descalzi, J.Martinez and S.Rica, Kluwer Academic Publ., Dordrecht, 2004, p. 33; preprint cond-mat/0209611

27. K.Nakazato and K.Kitahara, Prog. Theor. Phys. 64 (1980) 2261

28. R.A.Tahir-Kheli and R.J.Elliott, Phys. Rev. B 27 (1983) 844

29. K.W.Kehr and K.Binder, in: Application of the Monte Carlo Method in Statistical Physics, ed. K.Binder, Springer-Verlag, Berlin, 1987, and references therein.

30. M.-C.Desjonquéres and D.Spanjaard, Concepts in Surface Physics, Springer Verlag, Berlin, 1996

31. C.Landim, S.Olla and S.B.Volchan, Commun. Math. Phys. 192 (1998) 287

32. S.F.Burlatsky, G.Oshanin, A.M.Cazabat, M.Moreau and W.P.Reinhardt, Phys. Rev. E 54 (1996) 3892

33. O.Bénichou, A.M.Cazabat, A.Lemarchand, M.Moreau and G.Oshanin, J. Stat. Phys. 97 (1999) 351

34. P.Flajolet and A.M.Odlyzko, SIAM Journal on Discrete Math. 3 (1990) 216 